\title{
Generalized parton distributions and Deeply Virtual Compton Scattering on proton at CLAS
}

R. De Masi ${ }^{\text {* }}$

${ }^{a}$ DSM/DAPNIA/SPhN, CEA-Saclay,

91191 Gif-sur-Yvette, France

Two measurements of target and beam spin asymmetries for the reaction $e p \rightarrow e p \gamma$ were performed with CLAS at Jefferson Laboratory. Polarized $5.7 \mathrm{GeV}$ electrons were impinging on a longitudinally polarized ammonia and liquid hydrogen target respectively. These measurements are sensitive to Generalized Parton Distributions. Sizable $\sin \phi$ azimuthal angular dependences were observed in both experiments, indicating the dominance of leading twist terms and the possibility of extracting combinations of Generalized Parton Distributions on the nucleon.

\section{Introduction}

Generalized Parton Distributions (GPDs) are a very useful tool to describe the structure of hadrons at the quark-gluon level. They include as limiting case informations such as form factors and parton distributions, but they provide access also to unknown observables like the spatial distribution or the angular momentum of quarks and gluons inside the nucleon [1], [2].

GPDs can be accessed in hard exclusive processes. Among them, one of the cleanest for experimental investigation is the Deeply Virtual Compton Scattering (DVCS), a virtual photon impinging on a nucleon with production of a real photon in the final state and the nucleon staying intact. The high virtuality of the impinging photon $Q^{2}$ and the high energy transfer $\nu$ (Bjorken regime) allow the factorization of the process, that is the separation of the scattering amplitude in a hard scattering part (calculable in QED) and a nucleon part parametrized by GPDs, as shown in figure 1 .

However, the reaction $e p \rightarrow e p \gamma$ can also be due to the Bethe-Heitler process (BH), an ep scattering where the real photon in the final state is emitted by either the incident or the scattered leptons. DVCS and $\mathrm{BH}$ both contribute to the $e p \rightarrow e p \gamma$ scattering amplitude, with different

* On behalf on the CLAS Collaboration

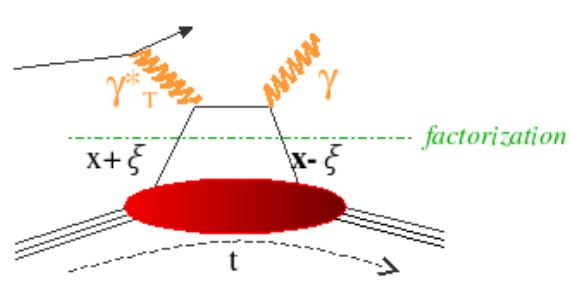

Figure 1. Feynman diagrams for DVCS showing the factorization

weights depending on the kinematics, like:

$T \sim\left|T_{D V C S}+T_{B H}\right|^{2}=\left|T_{D V C S}\right|^{2}+\left|T_{B H}\right|^{2}+I$

where $I$ is the DVCS-BH interference term.

Nevertheless, even in the regions where the BH is dominant, it is still possible to access the DVCS contribution via the interference term, by studying helicity dependent cross sections or asymmetries. In cross section differences, the helicityindependent $\mathrm{BH}$ term drops out; the interference of the two contributions enhances the effect of DVCS and produces sizeable cross section difference.

In this paper we show results for target spin asymmetry $\left(A_{U L}\right)$ and beam spin asymmetry $\left(A_{L U}\right)$, as obtained in the reaction $e p \rightarrow e p \gamma$ with 
CLAS. Being sensitive to different combinations of GPDs, the two asymmetries provide complementary informations.

The target spin asymmetry $A_{U L}$ is defined as

$A_{U L}=\frac{d \sigma^{\uparrow}(\phi)-d \sigma^{\downarrow}(\phi)}{d \sigma^{\uparrow}(\phi)+d \sigma^{\downarrow}(\phi)}$

where $\uparrow(\downarrow)$ represents the target polarization parallel (antiparallel) to the beam direction and $\phi$ is the angle between the leptonic and hadronic plane. At leading twist

$A_{U L} \propto\left\{F_{1} \tilde{\mathbf{H}}+\xi\left(F_{1}+F_{2}\right) \mathbf{H}+\ldots\right\} \sin \phi$

where $\xi=\frac{x_{B}}{2-x_{B}}, F_{1}$ and $F_{2}$ are the Dirac and Pauli proton form factors and $\tilde{\mathbf{H}}$ and $\mathbf{H}$ are sums over quark flavour of the corresponding GPDs at $x= \pm \xi$.

A similar definition holds for the beam spin asymmetry $A_{L U}$

$A_{L U}=\frac{d \sigma^{\uparrow}(\phi)-d \sigma^{\downarrow}(\phi)}{d \sigma^{\uparrow}(\phi)+d \sigma^{\downarrow}(\phi)}$

where $\uparrow(\downarrow)$ represents in this case the beam polarization parallel (antiparallel) to the beam direction. At leading twist

$A_{L U} \propto\left\{F_{1} \mathbf{H}+\ldots\right\} \sin \phi$

Explorative measurements of $A_{L U}$ have been performed by both CLAS [3] and HERMES [4] collaborations. Here we present a dedicated high statistics measurement which can study the dependence of $A_{L U}$ from $Q^{2}, x_{B}$ and $t$ in a large kinematical domain.

\section{The experimental set-up}

Both measurements discussed above used a $5.7 \mathrm{GeV}$ electron beam from the CEBAF accelerator and the CEBAF Large Acceptance Spectrometer (CLAS) [5].

CLAS is an almost $4 \pi$ magnetic spectrometer: it is equipped with drift chambers for track reconstruction, Cherenkov counters for electron identification, electromagnetic calorimeter (EC) for electrons and photons identification and scintillating counters for time-of-flight measurements.

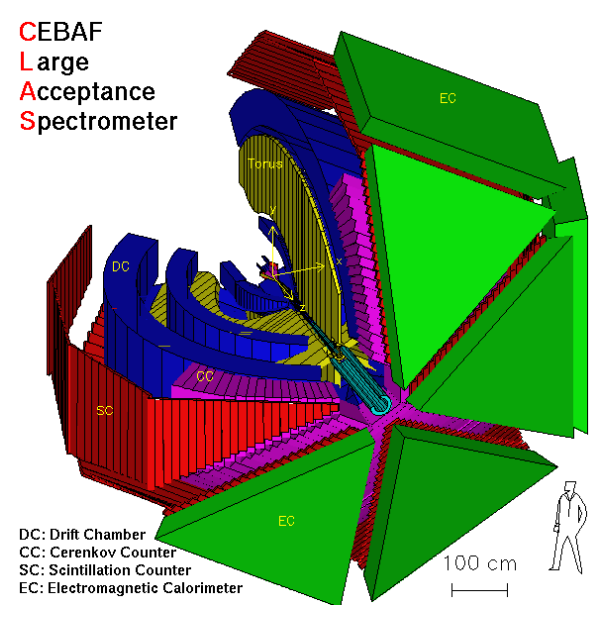

Figure 2. CEBAF Large Acceptance Spectrometer (CLAS)

The electron beam is longitudinally polarized $(P>75 \%)$. While beam polarization was mandatory for the $A_{L U}$ measurement, it was unnecessary for $A_{U L}$. The $A_{U L}$ asymmetry was averaged over the two beam helicities.

In case of the $A_{U L}$ measurement, the beam was impinging on a longitudinally polarized ${ }^{15} \mathrm{NH}_{3}$ target. The target polarization was insured by a solenoidal magnet with a $\delta B / B \sim 10^{-4}$ field homogeneity.

The elastic scattering of the beam on the atomic electrons causes the emission of so-called Møller electrons, with energies of the order of a few $\mathrm{MeV}$, which constitute the main background for the drift chambers. The target field insures also the focalisation along the beam line of the Møller electrons, reducing the background to a negligeable level.

In case of the $A_{L U}, \mathrm{LH}_{2}$ is used as target. In order to focus the Møller electrons a dedicated superconducting magnet was realized. No specific field homogeneity being required, the opening of the magnet was optimized to maximize the geometrical acceptance. Since the production cross section for DVCS photons peaks in the near forward direction, a dedicated electromagnetic 
calorimeter (IC, Inner Calorimeter) was built and installed to extend the polar angle coverage [7]. IC covers the polar angle range $5^{\circ}-16^{\circ}$, with full azimuthal coverage, while EC is used up to $40^{\circ}$ with partial azimuthal coverage. This guarantees the possibility to perform the measurement in a larger kinematical domain. The new IC was built of 424 small lead-tungstate crystals read out by avalanche photodiodes. It is the first calorimeter of this type to be used in a physics experiment. The calorimeter is shown in figure 3 : the crystals are clearly visible, while the avalanche photodiodes are mounted on the backplane.

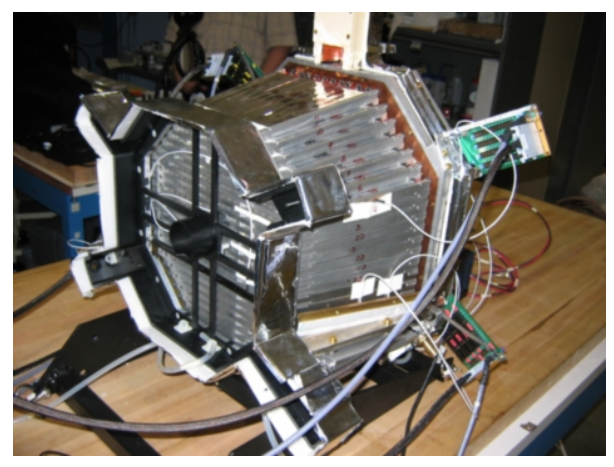

Figure 3. Inner Calorimeter IC

\section{Measurements}

In order to define deep inelastic kinematics, for both measurements presented in this work events were selected with $Q^{2}>1 \mathrm{GeV}^{2}$, and $W>2 \mathrm{GeV}$. The exclusive process $e p \rightarrow e p \gamma$ was determined by selecting all three particles in the final state. Cuts on missing energy and/or missing mass and on photon angles resulted in a clean identification of the process. The main background for the $e p \rightarrow e p \gamma$ process is $e p \rightarrow$ $e p \pi^{0}$ where the $\pi^{0}$ decays asymmetrically in two photons and the less energetic one is not detected. Even with tight kinematical cuts, such a contamination always occurs. It can be evaluated and subtracted using $e p \rightarrow e p \pi^{0}$ events unambiguously identified through 2-photons detection and normalized by a ratio of acceptances. For details on the analysis see [6] and [7].

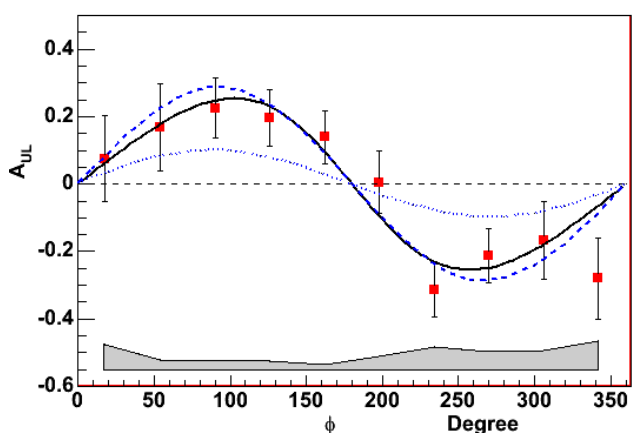

Figure 4. $A_{U L}$, for explication see text

Figure 4 shows the asymmetry $A_{U L}$ as function of the azimuthal angle $\phi$. Due to the limited statistics, the data were integrated on the whole $\left(Q^{2}, x_{B}, t\right)$ domain, where their average values are $\left\langle Q^{2}\right\rangle=1.82 \mathrm{GeV}^{2},\langle-t\rangle=0.31 \mathrm{GeV}^{2}$ and $\left\langle x_{B}\right\rangle=0.28$. The continous black curve is a fit of the data with a $\alpha \sin \phi+\beta \sin 2 \phi$ function. $\beta$ is consistent with 0 within the error bar, which indicates the dominance of leading twist terms. The dotted blue curve is a model prediction with $\tilde{H}=0$ while the dashed blue one is a full model prediction. This shows that $A_{U L}$ is sensitive to $\tilde{H}$ as previously discussed. The band at the bottom shows the systematical error.

A similar analysis is being performed for $A_{L U}$ for the dedicated 2005 run. This observable is sensitive to the GPD $H$. As mentioned above the high statistics and the increased acceptance will allow to map GPDs in a wide kinematical domain. The analysis is ongoing and preliminary results were showed at the conference.

From these results one infers that CLAS offers a excellent possibility for the study of GPDs. In the next future a dedicated experiment for the 
measurement of $A_{U L}$ is foreseen, to increase both the kinematical coverage (with IC) and the statistics. A second data taking is also foreseen for the $A_{L U}$ measurement which will double the present statistics. More data are still to come with the CLAS12 project: the upgraded CEBAF $12 \mathrm{GeV}$ electron beam and an upgraded CLAS detector will allow to extend the study of GPDs to a much larger kinematical domain as shown in figure 5 .

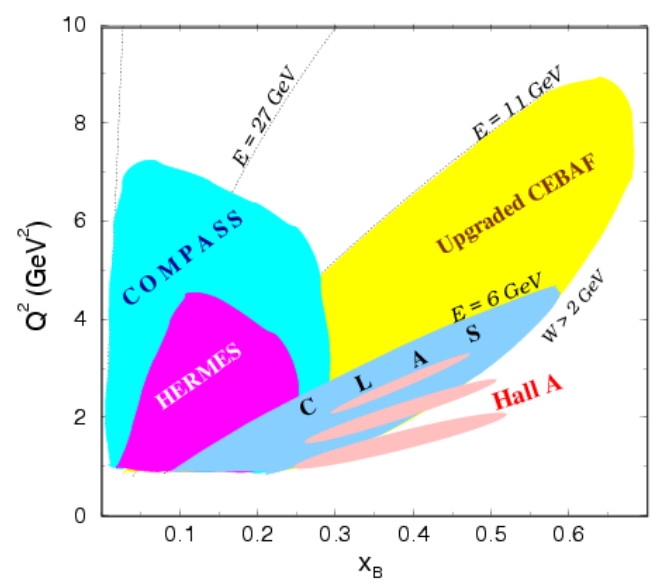

Figure 5. Kinematical domain of present and future experiments

\section{Summary}

We have presented two measurements of spin asymmetry for exclusive electroproduction of photons, as obtained with the CEBAF electron beam and CLAS. The $A_{U L}$ measurement shows its sensitivity to $\tilde{H}$, as expected from GPD models. The results of the $A_{L U}$ measurement will help constraint GPDs in a wide kinematical region. The large interest in this topic is shown by the number of experiment planned in the future, and theoretical interest as well, which will allow to characterize GPDs and to increase our knowledge on the hadron structure.

\section{REFERENCES}

1. X. Ji, Ann.Rev.Nucl.Part.Sci.54, 413 (2004).

2. A.V. Belitsky, A.V. Radyushkin, Phys.Rept. 418, 1 (2005).

3. S. Stepanyan et al., Phys. Rev. Lett. 87 182002 (2001).

4. A. Araipetian et al., Phys. Rev. Lett. 87 182001 (2001).

5. B. Mecking et al., Nucl. Instr and Meth. A 513, 503 (2003).

6. S. Chen et al., Phys. Rev. Lett. 97072002 (2006).

7. F.-X. Girod, Thèse de Doctorat, Université de Strasbourg, December 2006. 